\title{
KRICHEVER-NOVIKOV TYPE ALGEBRAS - PERSONAL RECOLLECTIONS OF JULIUS WESS
}

\author{
MARTIN SCHLICHENMAIER \\ University of Luxembourg, Mathematics Research Unit, FSTC \\ Campus Kirchberg, 6, rue Coudenhove-Kalergi \\ L-1359 Luxembourg-Kirchberg, Luxembourg \\ martin.schlichenmaier@uni.lu \\ Received 29 December 2011 \\ Revised 10 March 2012
}

\begin{abstract}
The author's research directions on Krichever-Novikov algebras was inspired by Julius Wess. After some recollections of Julius Wess, mainly from his Karlsruhe time, an overview over these (multi-point) algebras is given. They generalize the Witt, Virasoro, and related algebras to higher genus Riemann surfaces. Differential operator algebras, current algebras and their central extensions are discussed. For the two point situation some of these algebras were introduced by Krichever and Novikov in 1986. The multipoint generalization and the general setting is due to the author. References for further directions and applications are given.
\end{abstract}

Keywords: Krichever-Novikov type algebras; Witt algebra, Virasoro algebra, central extensions, Wess-Zumino-Novikov-Witten models; current algebras.

PACS numbers: 02.10.Hh, 01.60.+q, 03.65.Fd, 11.25-w

\section{Some Personal Recollections of Julius Wess}

This contribution starts with some rather personal remarks and private recollections of Julius Wess. The reader not interested in them should jump directly to Section 2 .

The intention of these remarks is not to present the scientific path of the author, but to show which a generous and inspiring person Julius was. Our contact begun when he was one of my academic teachers, evolved in time and we became friends up to the moment of his passing away. This is also true for his family. I remember well the discussions about literature which we had, the mutual recommendations for books to be read, the inspiring conversations on Robert Musil, Ingeborg Bachmann, but also about more profane things over a good meal and a good bottle of wine.

From 1978 till 1984 I studied mathematics at the University of Karlsruhe. As mathematics student one had to choose an application field (Nebenfach) to be studied beside it. I decided to go for Theoretical Physics. In this way I came into contact with Julius and his group. I heard several lecture courses about Quantum Field Theory with him. During this time Julius wished that the people in his group should 
learn about the mathematics related to anomalies. More precisely, he intended to start a joint seminar on Atiyah-Singer formula with somebody from the mathematics department. The analysis people in Karlsruhe were not interested in the subject, and the people from the algebra chair, where I had a student job as tutor (wissenschaftliche Hilfskraft), were totally overloaded by work. Julius approached me whether I would be willing to organize together with him on equal footing this seminar series. One could image how proud I was by this offer. In addition it fitted perfectly my interest in certain modern developments in mathematics. I developed a program, we discussed it, and got it started. I guess, Julius had underestimated the necessary mathematical background to be covered before we could even reach the point when we could formulate the Atiyah-Singer formula. But he insisted that we should try to head for a thorough mathematical treatment. It was always his goal that his students should have an excellent mathematical background. They should not just use it as a black box, but understand the mathematics they were using. In this way I was accepted at the famous 12th floor of the Physik-Hochhaus in Karlsruhe.

In 1984, roughly one year later, after finishing my diploma thesis and all the exams I went with a fellowship of the DAAD to the Brandeis University, Massachusetts, USA. In the seminar, we had not yet reached the final stage. But we got to the Sobolev theory part, and Dan Socolescu, a mathematician working in analysis in Karlsruhe, took care of it at the seminar.

In fall 1985, I returned back to Germany and found a position as wissenschaftlicher Mitarbeiter (assistant) at the Mathematics Department of the University of Mannheim. Immediately, Julius reestablished the contact. He had received the Leibniz-Prize in 1986. It was well-endowed with money, which he could spent in the coming years. He offered me that I should come every Friday to Karlsruhe (Mannheim is only $60 \mathrm{~km}$ away) to bring the seminar to an end and to participate as a mathematician in the scientific life of the Institute of Theoretical Physics. With pleasure I agreed as for my scientific work the atmosphere there was very inspiring. We could finish within one year the Atiyah-Singer theorem, including a number of important applications.

This was the time when string theory had his revival. Julius asked me to give a lecture course about Riemann surfaces (they appeared as world sheets in this version of the theory). I gave the course and then he convinced me that a write-up of them, published in the Springer Lecture Notes Series, would be highly welcome. It appeared in 1989, see Ref. 1. Would I have known how much work it was to polish everything to be suitable for a publications, I guess I would have refused it. But now it was done, and I have to say, that it was quite much in demand. It was reprinted several times unchanged. In 2007, Springer approached me and a second quite enlarged edition appeared again with Springer, now in the Theoretical and Mathematical Physics series, see Ref. 2.

During my stay at Brandeis, I started to think about a possible topic for my $\mathrm{PhD}$ thesis. I got interested in the explicit construction of the moduli space of stable 
rank two vector bundles over elliptic surfaces, i.e. over complex two-dimensional manifolds which admit a fibering by elliptic curves, resp. one-dimensional complex tori. After a certain success I got stuck and could not advance anymore in direction of my final goal. Frustration started. In fact, the problem is still not solved today.

But by chance Julius came as rescue. He showed me some articles ${ }^{3}$ of Krichever and Novikov which the authors had given to him. There they present some parts of a global operator approach to string theory and conformal field theory on higher genus Riemann surfaces. He asked me whether I could explain him the mathematics behind the articles. I have to admit it was not an easy reading. But I managed to get the main points which I could explain to him. During this undertaking, I realized that for the intended application they had in mind the two point case which they considered was not enough. $^{\text {a }}$ One has to generalize it to more points. This is not at all just a straightforward generalization. But quite fast I managed the problems, introduced the objects, constructed representations, etc. These are now the multipoint Krichever-Novikov type algebras which I present in the other sections of this contribution. Within one year I had prepared a text which I considered to be valid for a PhD thesis (a Dissertation in German). I asked Rainer Weissauer (Mannheim) whether he would be willing to accept it and I was happy that he agreed. Finally, in 1990 I got my PhD in mathematics from the University of Mannheim. ${ }^{4}$

Julius Wess never published something on Krichever-Novikov type algebras himself. But in his group some students and postdocs worked on the subject. To mention some names Thomas Deck, ${ }^{5}$ A. Anzaldo-Meneses, ${ }^{6}$ and Andreas Ruffing. ${ }^{7}$ Diploma thesis were produced, some of them under my guidance. Also on the famous Bayrischzell meetings, which just started, the subject was discussed.

By Julius approaching me about explaining a paper to him, one of my still active research directions in mathematics had started. As a remark aside, in joint collaboration with Oleg Sheinman I applied this techniques in the context of a global operator approach to Wess-Zumino-Novikov-Witten models. ${ }^{8,9,10}$

As already written above, the scientific atmosphere in the 12th floor was extremely inspiring. Julius invited quite a number of people of very different background and interest. For examples during these days I met Jens Hoppe and Peter Schaller there. They worked in membrane theory and wanted to understand $S U(N), N \rightarrow \infty$ limits. There was quite a lot of misconception in the physics literature. We met Martin Bordemann from Freiburg, he joint us and we wrote a first paper $^{11}$ together in 1990 . We formulated the conjecture that by geometric quantization for Kähler manifolds the Poisson algebra is in a certain sense always such a (weak) limit. Later in 1993 together with Martin Bordemann and Eckhard Meinrenken we could indeed show this. ${ }^{12}$ We showed the even more important statement, that the Berezin-Toeplitz quantization scheme has the correct semi-classical limit,

a This is again an example that it is always helpful to explain a complicated subject to somebody else. One always will get some new insight and ideas. 
from which our conjecture follows. Different aspects of Berezin-Toeplitz quantization $^{13}$ is my second research direction; again inspired by the working group of Julius Wess in Karlsruhe.

Later Julius moved to Munich to the LMU and the MPI (Heisenberg Institute). Hence, weekly contact was not possible anymore. But as he said, I always had an invitation for one month per year to come to Munich. Unfortunately, I could not always profit from this, as I had also my usual duties at the University of Mannheim. But when I was there we discussed about algebraic geometry, quantum groups, Hopf algebras, noncommutative geometry, star products and many more things. Let me point out that star products also appear in my work on Berezin Toeplitz quantization.

Even if we never wrote a joint paper (I guess my style as mathematician and his as a physicist were too different), our discussions were fruitful in both directions.

Together with Alice Fialowski, Marc de Montigny, and Sergey Novikov I organized at the Mathematisches Forschungsinstitut Oberwolfach (MFO) in 2006 a workshop on Deformations and Contractions in Mathematics and Physics. ${ }^{14}$ Of course, Julius was invited and he enjoyed his participation. Alice and I decided to apply for some kind of follow-up workshop again in Oberwolfach and asked Jürg Fröhlich and Julius Wess to join the organization team. Also Julius immediately said yes and contributed with a number of helpful suggestions. On July 27, 2007, I finalized the application, submitted it and send him an email informing him about the submission. Then for three weeks I left for summer vacation. When I returned I found several messages of Paolo Aschieri on my answering machine. He tried to contact me several times, first telling me that Julius had a stroke and that was in hospital under very critical conditions, then that he died on August 8, 2007, and finally when the funeral was. But I was too late.

I have to mourn a friend and an academic teacher. Let me add that the proposal for the workshop was approved and took place in $2010 .{ }^{15}$ For sure, every participants missed him there, but in thoughts he was with us.

\section{From Virasoro Algebra to Krichever - Novikov Algebras}

Lie groups and Lie algebras are related to symmetries of systems. By the use of the symmetry the system can be better understood, may be it is even possible to solve it in a certain sense. Here we deal with systems which have an infinite number of independent degrees of freedom. They appear for example in Conformal Field Theory (CFT), see Refs. 16, 17. But also in the theory of partial differential equations and at many other places in- and outside of mathematics they play an important role. The appearing Lie groups and Lie algebras are infinite dimensional. Some of the simplest nontrivial infinite dimensional Lie algebras are the Witt algebra and its central extension the Virasoro algebra. We will recall their definition in the following. In the sense explained below (in particular in CFT) they are related to what is called the genus zero situation. For CFT on higher genus Riemann surfaces 
the Krichever-Novikov type algebras, to be discussed here, will show up as algebras of global symmetry operators.

The Witt algebra ${ }^{\mathrm{b}} \mathcal{W}$ is the Lie algebra generated as vector space by the elements $\left\{e_{n} \mid n \in \mathbb{Z}\right\}$ with Lie structure

$$
\left[e_{n}, e_{m}\right]=(m-n) e_{n+m}, \quad n, m \in \mathbb{Z} .
$$

One of its realization is as complexification of the Lie algebra of polynomial vector fields $V e c t_{\text {pol }}\left(S^{1}\right)$ on the circle $S^{1}$, which is a subalgebra of $\operatorname{Vect}\left(S^{1}\right)$, die Lie algebra of all $C^{\infty}$ vector fields on the circle. In this realization $e_{n}=-\mathrm{i} \exp (\mathrm{i} n \varphi) \frac{d}{d \varphi}$. The Lie product is the usual Lie bracket of vector fields.

If we extend these generators to the whole punctured complex plane we obtain $e_{n}=z^{n+1} \frac{d}{d z}$. This gives another realization of the Witt algebra as the algebra of meromorphic vector fields on the Riemann sphere $\mathbb{P}^{1}(\mathbb{C})$ which are holomorphic outside $\{0\}$ and $\{\infty\}$. This will be our starting point for generalizations.

A very important fact is that the Witt algebra is a $\mathbb{Z}$-graded Lie algebra. We define the degree by setting $\operatorname{deg}\left(e_{n}\right):=n$. The Lie product between elements of degree $n$ and of degree $m$ is of degree $n+m$ (if nonzero). The homogeneous spaces $\mathcal{W}_{n}$ of degree $n$ are one-dimensional with basis $e_{n}$.

In the process of quantizing, or regularization one is often forced to modify an action of a Lie algebra. A typical example is given by the product of infinite sums of operators. Quite often they are only well-defined if a certain "normal ordering" is introduced. In this way the modified action will only be a projective action. This can be made to an honest Lie action by passing to a suitable central extension of the Lie algebra.

For the Witt algebra the universal one-dimensional central extension is the Virasoro algebra $\mathcal{V}$. As vector space it is the direct $\operatorname{sum} \mathcal{V}=\mathbb{C} \oplus \mathcal{W}$. If we set for $x \in \mathcal{W}$, $\hat{x}:=(0, x)$, and $t:=(1,0)$ then its basis elements are $\hat{e}_{n}, n \in \mathbb{Z}$ and $t$ with the Lie product

$$
\left[\hat{e}_{n}, \hat{e}_{m}\right]=(m-n) \hat{e}_{n+m}-\frac{1}{12}\left(n^{3}-n\right) \delta_{n}^{-m} t, \quad\left[\hat{e}_{n}, t\right]=[t, t]=0,
$$

for all $n, m \in \mathbb{Z}^{\mathrm{c}}$. If we set $\operatorname{deg}\left(\hat{e}_{n}\right):=\operatorname{deg}\left(e_{n}\right)=n$ and $\operatorname{deg}(t):=0$ then $\mathcal{V}$ becomes a graded algebra. The algebra $\mathcal{W}$ will only be a subspace, not a subalgebra of $\mathcal{V}$. But it will be a quotient. In some abuse of notation we identify the element $\hat{x} \in \mathcal{V}$ with $x \in \mathcal{W}$. Up to equivalence and rescaling the central element $t$, this is beside the trivial (splitting) central extension the only central extension.

The above is the genus zero situation, i.e. where we consider the Riemann sphere, and our objects are allowed to have poles at the two points $\{0, \infty\}$. From the application in CFT it is completely natural to look for generalizations to (compact) Riemann surfaces of arbitrary genus. Also we will need to allow poles at a finite, but

\footnotetext{
${ }^{\mathrm{b}}$ Also sometimes called Virasoro algebra without central term.

${ }^{c}$ Here $\delta_{k}^{l}$ is the Kronecker delta which is equal to 1 if $k=l$, otherwise zero.
} 


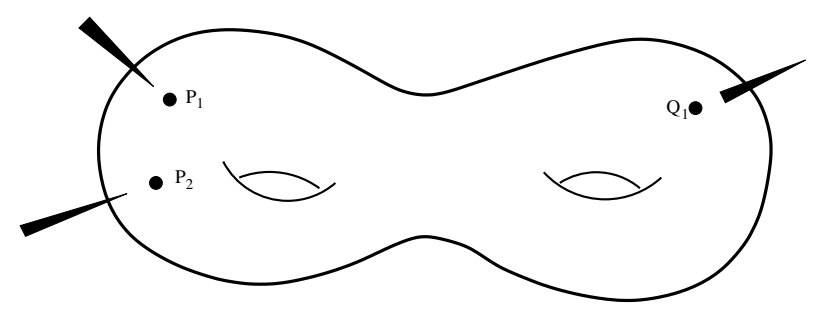

Fig. 1. Riemann surface of genus two with two incoming and one outgoing point.

arbitrary number of marked points. In the interpretation of CFT they correspond to incoming fields and outgoing fields. Hence, the set of marked points will have a natural decomposition into two disjoint subsets.

More precisely, let $M$ be a compact Riemann surface of genus $g$, and $N, K \in \mathbb{N}$ with $N \geq 2$ and $1 \leq K<N$. Fix

$$
I=\left(P_{1}, \ldots, P_{K}\right), \quad \text { and } \quad O=\left(Q_{1}, \ldots, Q_{N-K}\right)
$$

disjoint ordered tuples of distinct points ("marked points", "punctures") on the Riemann surface. In particular, we assume $P_{i} \neq Q_{j}$ for every pair $(i, j)$. The points in $I$ are called the in-points, the points in $O$ the out-points. Sometimes we consider $I$ and $O$ simply as sets and set $A=I \cup O$ as a set. In Figure 1 the case of a Riemann surface of genus 2 with two incoming points and one outgoing point is visualized.

Our global objects, algebras, structures, ... will be meromorphic objects which are holomorphic outside of the points in $A$.

The grading of the Witt and Virasoro algebra was very important for the construction of highest weight representations. For our generalized situation, we do not have an honest grading, but only an almost-grading (its definition will be given below). It was realized by Krichever and Novikov ${ }^{3}$ that this will still suffice for this purpose. The almost-grading will be induced by the splitting of $A$ into $I$ and $O$. They introduced it in the case where $A$ has only two points. In this case the splitting is uniquely given. I gave the generalization to arbitrary (finite) sets $A$ and splittings in Refs. 18, 19, 20, 4. In the case of two points my description gives back the Krichever-Novikov description. If one specializes even more to $g=0$ and $A$ consisting of two points, which we might assume to be $\{0\}$ and $\{\infty\}$, i.e.

$$
M=\mathbb{P}^{1}(\mathbb{C})=S^{2}, \quad I=\{z=0\}, \quad O=\{z=\infty\}
$$

then we obtain back the well-known algebras of Conformal Field Theory (CFT), Witt, Virasoro, affine, etc. Under "classical situation" we will understand in the following always this geometric situation.

\section{Krichever-Novikov Algebras}

Let $M, A=I \cup O$ as above. In view of the representations to be discussed later, let us be a little bit more general. Let $\mathcal{F}^{\lambda}=\mathcal{F}^{\lambda}(A)$ be the infinite dimensional vector 
space of meromorphic differential forms of weight $\lambda$ which are holomorphic outside of $A$. In a local holomorphic coordinate $z$ they look like $f_{\mid}=\hat{f}(z)(d z)^{\lambda}$ with $\hat{f}(z)$ a local meromorphic function. In this review we will only consider $\lambda \in \mathbb{Z}$. But after the choice of a "square root" of the differentials, i.e. a form of weight $1 / 2$, we might allow $\lambda \in \frac{1}{2} \mathbb{Z}$. Indeed, with them higher genus generalizations of the Neveu-Schwarz and Ramond supersymmetric algebras can be given. Special cases of the weights are the functions $(\lambda=0)$, the space is also denoted by $\mathcal{A}$, the vector fields $(\lambda=-1)$, denoted by $\mathcal{L}$, the differentials $(\lambda=1)$, and the quadratic differentials $(\lambda=2)$.

The space $\mathcal{A}$ becomes by pointwise multiplication an associative commutative algebra. In the classical situation it is the algebra $\mathbb{C}\left[z, z^{-1}\right]$ of Laurent polynomials. The spaces $\mathcal{F}^{\lambda}$ are always modules over $\mathcal{A}$.

The space $\mathcal{L}$ as space of vector fields is equipped with the Lie bracket [.,.] of vector fields. Its local form is ${ }^{\mathrm{d}}$

$$
[e, f]_{\mid}=\left[e(z) \frac{d}{d z}, f(z) \frac{d}{d z}\right]:=\left(e(z) \frac{d f}{d z}(z)-f(z) \frac{d e}{d z}(z)\right) \frac{d}{d z} .
$$

By taking the Lie derivative the vector spaces $\mathcal{F}^{\lambda}$ become Lie modules over $\mathcal{L}$.

As the elements of $\mathcal{L}$ are acting as derivations on $\mathcal{A}$ we can define the Lie algebra of differential operators of degree $\leq 1$, denoted by $\mathcal{D}^{1}$, as the semi-direct sum of the abelian Lie algebra $\mathcal{A}$ with $\mathcal{L}$. Its universal enveloping algebra will be the algebra of all differential operators of arbitrary degree. For more details see Refs. 4, 21, 22.

The next object which we have to generalize is the classical current algebra to the higher-genus, multi-point current algebra of Krichever-Novikov type. We start with $\mathfrak{g}$ a complex finite-dimensional Lie algebra and endow the tensor product $\overline{\mathfrak{g}}=\mathfrak{g} \otimes_{\mathbb{C}} \mathcal{A}$ with the Lie bracket

$$
[x \otimes f, y \otimes g]=[x, y] \otimes f \cdot g, \quad x, y \in \mathfrak{g}, \quad f, g \in \mathcal{A} .
$$

The algebra $\overline{\mathfrak{g}}$ is the higher genus current algebra. It is an infinite dimensional Lie algebra and might be considered as the Lie algebra of $\mathfrak{g}$-valued meromorphic functions on the Riemann surface with poles only outside of $A$. Note that we allow also the case of $\mathfrak{g}$ an abelian Lie algebra. In the classical case the current algebra $\overline{\mathfrak{g}}$ is the standard current algebra $\overline{\mathfrak{g}}=\mathfrak{g} \otimes \mathbb{C}\left[z^{-1}, z\right]$ with Lie bracket

$$
\left[x \otimes z^{n}, y \otimes z^{m}\right]=[x, y] \otimes z^{n+m} \quad x, y \in \mathfrak{g}, \quad n, m \in \mathbb{Z} .
$$

\section{Almost-Graded Structure}

As already mentioned above for constructions of certain type of representations, like highest weight representations, vacuum type representations, etc. a grading or at least some substitute is needed. In Refs. 4, 20 for all above mentioned algebras and modules an almost-graded structure is introduced like described in the following.

\footnotetext{
${ }^{\mathrm{d}}$ To avoid cumbersome notation we will quite often use the same symbol for the form and its representing function.
} 
For $\mathcal{F}^{\lambda}$ we introduce subspaces $\mathcal{F}_{m}^{\lambda}$ of dimension $K=\# I$ by exhibiting certain elements $f_{m, p}^{\lambda} \in \mathcal{F}^{\lambda}, p=1, \ldots, K$ which constitute a basis of $\mathcal{F}_{m}^{\lambda}$. These elements are called elements of degree $m$, the subspace is called homogeneous subspace of degree $m$. The degree is essentially given by the zero orders of the elements at the points in $I$. To get an impression of the kind of prescription let me give it for $I=\left\{P_{1}, \ldots, P_{K}\right\}$ and $O=\{Q\}$ which is an important special case anyhow

$$
\begin{aligned}
\operatorname{ord}_{P_{i}}\left(f_{m, p}^{\lambda}\right) & =(n+1-\lambda)-\delta_{i}^{p}, \quad i=1, \ldots, K \\
\operatorname{ord}_{Q}\left(f_{m, p}^{\lambda}\right) & =-K \cdot(n+1-\lambda)+(2 \lambda-1)(g-1) .
\end{aligned}
$$

The description for the points in $I$ is always valid (even if $O$ has more than one points). The description at $Q$ is valid for $g=0$ and for $g \geq 2, \lambda \neq 0,1$ if the points of $A$ are in generic position. In the other cases the description at $Q$ has to be adapted to make the element $f_{n, p}^{\lambda}$ unique up to rescaling by a nonvanishing constant. For $\lambda=0$ or $\lambda=1$, resp. for $g=1$ we have to take into account also duality with respect to Krichever-Novikov pairing introduced in Section 5.1. The proof of the existence $^{4,18}$ is based on the Theorem of Riemann-Roch. ${ }^{2}$

Theorem 4.1. The algebras $\mathcal{A}, \mathcal{L}, \mathcal{D}^{1}$ are almost-graded associative, resp. Lie algebras and the modules $\mathcal{F}^{\lambda}$ are almost-graded modules over them. The almostgrading depends on the splitting of the set $A$ into $I$ and 0.

This means the following: We have that $\mathcal{F}^{\lambda}$ is the direct sum of the finitedimensional subspace $\mathcal{F}_{m}^{\lambda}$

$$
\mathcal{F}^{\lambda}=\bigoplus_{m \in \mathbb{Z}} \mathcal{F}_{m}^{\lambda}, \quad \text { with } \quad \operatorname{dim} \mathcal{F}_{m}^{\lambda}=K
$$

Furthermore, there exists $S_{1}, S_{2}, S_{3}, \ldots$ (independent of $n$ and $m$ ) such that

$$
\mathcal{A}_{n} \cdot \mathcal{A}_{m} \subseteq \bigoplus_{h=n+m}^{n+m+S_{1}} \mathcal{A}_{h}, \quad\left[\mathcal{L}_{n}, \mathcal{L}_{m}\right] \subseteq \bigoplus_{h=n+m}^{n+m+S_{2}} \mathcal{L}_{h}, \quad\left[\mathcal{D}_{n}^{1}, \mathcal{D}_{m}^{1}\right] \subseteq \bigoplus_{h=n+m}^{n+m+S_{3}} \mathcal{D}_{h}^{1}
$$

and

$$
\mathcal{A}_{n} \cdot \mathcal{F}_{m}^{\lambda} \subseteq \bigoplus_{h=n+m}^{n+m+S_{4}} \mathcal{F}_{h}^{\lambda}, \quad \mathcal{L}_{n} \cdot \mathcal{F}_{m}^{\lambda} \subseteq \bigoplus_{h=n+m}^{n+m+S_{5}} \mathcal{F}_{h}^{\lambda}, \quad \mathcal{D}_{n}^{1} \cdot \mathcal{F}_{m}^{\lambda} \subseteq \bigoplus_{h=n+m}^{n+m+S_{6}} \mathcal{F}_{h}^{\lambda} .
$$

For the proof of this theorem see Refs. 4, 20.

In contrast to a honest grading we have an uniform finite band-width around the ideal value, which is the sum of the degrees. The constants $S_{i}$ depend on the genus of the Riemann surface, the number of points in $I$ and $O$ and the weight $\lambda$. In fact they can be explicitly calculated and this is done in Ref. 4 . In the two-point generic case we obtain for the function algebra $\mathcal{A}$ the value $S_{1}=g$, and for the vector field algebra $\mathcal{L}$ the value $S_{2}=3 \mathrm{~g}$. Obviously, for the classical situation $S_{1}=S_{2}=0$ (in fact all $\left.S_{i}=0\right)$. Hence, we get an honest grading.

As $\overline{\mathfrak{g}}=\mathfrak{g} \otimes \mathcal{A}$ the almost-grading of $\mathcal{A}=\oplus \mathcal{A}_{m}$ induces an almost-grading $\overline{\mathfrak{g}}=\oplus \overline{\mathfrak{g}}_{m}$ with $\overline{\mathfrak{g}}_{m}=\mathfrak{g} \otimes \mathcal{A}_{m}$. 
On the basis of the almost-grading we can give a triangular decomposition of the algebras. In fact it induces in each case the existence of two infinite dimensional subalgebras and in between a finite-dimensional subspace (we call it the critical strip). For example

$$
\mathcal{L}=\mathcal{L}_{[+]} \oplus \mathcal{L}_{[0]} \oplus \mathcal{L}_{[-]} \quad \mathcal{A}=\mathcal{A}_{[+]} \oplus \mathcal{A}_{[0]} \oplus \mathcal{A}_{[-]} \quad \overline{\mathfrak{g}}=\overline{\mathfrak{g}}_{[+]} \oplus \overline{\mathfrak{g}}_{[0]} \oplus \overline{\mathfrak{g}}_{[-]} .
$$

where

$$
\mathcal{L}_{[+]}:=\bigoplus_{m>0} \mathcal{L}_{m}, \quad \mathcal{L}_{[-]}:=\bigoplus_{m<-S_{2}} \mathcal{L}_{m}, \quad \mathcal{L}_{[0]}=\bigoplus_{m=-S_{2}}^{m=0} \mathcal{L}_{m} .
$$

Similar for $\mathcal{A}$ and $\overline{\mathfrak{g}}$. By the almost-gradednes the $[+]$ and $[-]$ subspaces are indeed subalgebras. The [0] spaces in general not. Only for the classical situation they are one-dimensional and subalgebras too.

\section{Central Extensions}

\subsection{Separating cycle and Krichever-Novikov duality}

Let $C_{S}$ be a smooth 1-cycle on the Riemann surface $M$, i.e. a collection of smooth connected curves. All curves should be positively oriented (with respect to the orientation of $M$ ) and should be counted with multiplicity one. Furthermore, the curve should separate the points in $I$ from the points in $O$. This means if we cut the surface along $C_{S}$ it should decompose into two (not-necessarily connected) pieces which by the orientation can be considered as the inside and the outside. All points in $I$ should lie in the inside, all points in $O$ should lie an the outside. Such a cycle is called separating cycle. Examples are given by a collection of positive oriented circles around each of the points in $I$, or by a collection of negative oriented circles around the points in $O$. In Figure 1 also the "waist" between the two "holes" is of this type. A non-example is the loop going around one of the branches in the hole. Examples are also given as level lines of a "proper time evolution", for which I refer to Ref. 20.

Given such a separating cycle we can define a linear map

$$
\mathcal{F}^{1} \rightarrow \mathbb{C}, \quad \omega \mapsto \frac{1}{2 \pi \mathrm{i}} \int_{C_{S}} \omega
$$

This map will not depend on the separating line $C_{S}$ chosen, as two of such will be homologous and the poles of $\omega$ are only located in $I$ and $O$. This map will play a role for defining cocycles in Section 5.3. Note also that the integration of $\omega$ over $C_{S}$ can be described over special cycles which are either circles around the points in $I$, or around the points in $O$. But this corresponds to calculating residues

$$
\omega \mapsto \frac{1}{2 \pi \mathrm{i}} \int_{C_{S}} \omega=\sum_{k=i}^{K} \operatorname{res}_{P_{i}}(\omega)=-\sum_{l=1}^{N-K} \operatorname{res}_{Q_{l}}(\omega) .
$$


Furthermore,

$$
\mathcal{F}^{\lambda} \times \mathcal{F}^{1-\lambda} \rightarrow \mathbb{C}, \quad(f, g) \mapsto\langle f, g\rangle:=\frac{1}{2 \pi \mathrm{i}} \int_{C_{S}} f \cdot g,
$$

gives a well-defined pairing, called the Krichever-Novikov (KN) pairing. The basis $\left\{f_{n, p}^{\lambda}\right\}$ and $\left\{g_{-m, p}^{1-\lambda}\right\}$ will be a dual basis with respect to the KN pairing, i.e.

$$
\left\langle f_{n, p}^{\lambda}, g_{-m, r}^{1-\lambda}\right\rangle=\delta_{p}^{r} \delta_{n}^{m} .
$$

From this it follows that (13) is non-degenerate.

\subsection{Central extensions and cocycles}

A central extension of the Witt algebra is the Virasoro algebra. As it is written in (2) there is no natural way to extend it to higher genus. First we have to geometrize it. Let me recall that a central extension of a Lie algebra $W$ is given as vector space direct sum $\widehat{W}=\mathbb{C} \oplus W$. If we denote $\hat{x}:=(0, x)$ and $t:=(1,0)$ then the Lie structure is given by

$$
[\hat{x}, \hat{y}]=\widehat{[x, y]}+\psi(x, y) \cdot t, \quad[t, \widehat{W}]=0, \quad x, y \in W .
$$

$\widehat{W}$ will be a Lie algebra, e.g. fulfill the Jacobi identity, if and only if $\psi$ is antisymmetric and fulfills the Lie algebra 2-cocycle condition

$$
0=d_{2} \psi(x, y, z):=\psi([x, y], z)+\psi([y, z], x)+\psi([z, x], y) .
$$

There is the notion of equivalence of central extensions. It turns out that two central extensions are equivalent if and only if the difference of their defining 2-cocycles $\psi$ and $\psi^{\prime}$ is a coboundary, i.e. there exists a $\phi: W \rightarrow \mathbb{C}$ such that

$$
\psi(x, y)-\psi^{\prime}(x, y)=\phi([x, y]) .
$$

In this way the second Lie algebra cohomology $\mathrm{H}^{2}(W, \mathbb{C})$ of $W$ with values in the trivial module $\mathbb{C}$ classifies equivalence classes of central extensions. The class [0] corresponds to the trivial (i.e. splitting) central extension. Hence, to construct central extensions of our algebras we have to find such Lie algebra 2-cocycles.

\subsection{Geometric Cocycles}

In the following we will give geometrically defined 2-cocycles for our algebras.

\subsubsection{The function algebra $\mathcal{A}$}

We consider it as abelian Lie algebra. Let $C$ be an arbitrary smooth but not necessarily connected curve. We set

$$
\psi_{C}^{1}(g, h):=\frac{1}{2 \pi \mathrm{i}} \int_{C} g d h, \quad g, h \in \mathcal{A} .
$$




\subsubsection{The current algebra $\overline{\mathfrak{g}}$}

For $\overline{\mathfrak{g}}=\mathfrak{g} \otimes \mathcal{A}$ we first have to fix $\beta$ a symmetric, invariant, bilinear form on $\mathfrak{g}$ (not necessarily non-degenerate). Invariance means that we have $\beta([x, y], z)=\beta(x,[y, z])$ for all $x, y, z \in \mathfrak{g}$. The cocycle is now given as

$$
\psi_{\beta, C}^{2}(x \otimes g, y \otimes h):=\beta(x, y) \cdot \frac{1}{2 \pi \mathrm{i}} \int_{C} g d h, \quad x, y \in \mathfrak{g}, g, h \in \mathcal{A} .
$$

\subsubsection{The vector field algebra $\mathcal{L}$}

Here it is a little bit more delicate. First we have to choose a (holomorphic) projective connection $R$, see the appendix for its definition. We define

$$
\psi_{C, R}^{3}(e, f):=\frac{1}{24 \pi \mathrm{i}} \int_{C}\left(\frac{1}{2}\left(e^{\prime \prime \prime} f-e f^{\prime \prime \prime}\right)-R \cdot\left(e^{\prime} f-e f^{\prime}\right)\right) d z .
$$

Only by the term related with the projective connection it will be a well-defined differential, i.e. independent of the coordinate chosen. It is shown in Ref. 4 that it is a cocycle. Another choice of a projective connection will result in a cohomologous one.

\subsubsection{The differential operator algebra $\mathcal{D}^{1}$}

For the differential operator algebra the cocycle for $\mathcal{A}$ can be extended by zero on the subspace $\mathcal{L}$ and the cocycles for $\mathcal{L}$ can be pulled back. In addition there is a third type of cocycles mixing $\mathcal{A}$ and $\mathcal{L}$ :

$$
\psi_{C, T}^{4}(e, g):=\frac{1}{24 \pi \mathrm{i}} \int_{C}\left(e g^{\prime \prime}+T e g^{\prime}\right) d z, \quad e \in \mathcal{L}, g \in \mathcal{A},
$$

with an affine connection $T$ (see the appendix for its definition). For more details on the cocycles, see Ref. 22 .

\subsection{Uniqueness and classification of central extensions}

In contrast to the classical situation there are many essentially different closed curves and also many non-equivalent central extensions defined by the integration.

But we should take into account that we want to extend the almost-grading from our algebras to the centrally extended ones. This means we take $\operatorname{deg} \hat{x}:=\operatorname{deg} x$ and assign a degree $\operatorname{deg}(t)$ to the central element $t$, and still have the almost-gradedness.

This is possible if and only if our defining cocycle $\psi$ is "local" in the following sense (the name was introduced in the two point case by Krichever and Novikov in Ref. 3). There exists an $M_{1}, M_{2} \in \mathbb{Z}$ such that

$$
\forall n, m: \psi\left(W_{n}, W_{m}\right) \neq 0 \Longrightarrow M_{1} \leq n+m \leq M_{2} .
$$

Here $W$ stands for any of our algebras. Very important, "local" is defined in terms of the grading, and the grading itself depends on the splitting $A=I \cup O$, hence what is "local" depends on the splitting too. 
If we choose as integration path a separating cocycle $C_{S}$ then the above introduced geometric cocycles are local. Recall that in this case integration can be done by calculating residues at the in-points or at the out-points. All these cocycles are cohomologically nontrivial.

The question is: Is the opposite also true? This means is an arbitrary local cocycle cohomologous to the geometric cocycles with integration over $C_{S}$ introduced above. The answer is essentially yes. This means it is true how far it can be expected. The precise results and proofs are done in Ref. 22 for the vector field, function and differential operator algebras and in Ref. 23 for the current algebras.

In particular, for the vector field algebra there is a unique cocycle class which contains all local cocycles. A representing one can be given by (20) integrated over a $C_{S}$. For the function algebra one has to add the condition of $\mathcal{L}$-invariance, i.e.

$$
\psi(e . g, h)+\psi(g, e . h)=0, \quad \forall e \in \mathcal{L}, g, h \in \mathcal{A}
$$

to obtain uniqueness. For the differential operator algebra $\mathcal{D}^{1}$ the subspace of local classes is 3 -dimensional, generated by the cocycle for $\mathcal{L}(20), \mathcal{A}(18)$ and the mixing cocycle (21), all of them integrated over a separating cycle $C_{S}$.

For the current algebra with $\mathfrak{g}$ a finite dimensional simple Lie algebra we have again uniqueness of local cocycle classes. For $\mathfrak{g}$ a reductive Lie algebra and the cocycle $\mathcal{L}$-invariant if restricted to the abelian part, a complete classification of local cocycle classes can be found in Ref. 23. Note that in the case of a simple Lie algebra every symmetric, invariant bilinear form $\beta$ is a multiple of the Cartan-Killing form.

I like to mention that in all the applications I know of, the cocycle coming from representations, regularizations, etc. is local, hence, the uniqueness or classification can be used.

\section{Further developments and applications}

Above I introduced the basic concepts, results about the structure of these Krichever-Novikov type algebras and their central extensions. Of course, this does not close the story. But due to space limitations I will only be able to name certain important additional constructions, like the construction of representations of certain type and their applications. I will give additional references for further study.

\subsection{Semi-infinite forms and fermionic Fock space representations}

Our Krichever-Novikov vector field algebras $\mathcal{L}$ have as Lie modules the spaces $\mathcal{F}^{\lambda}$. But these representations are not of the type one is usually interested in, there is no ground state (no vacuum). Using the grading of $\mathcal{F}^{\lambda}$ it is possible to construct semi-infinite wedge forms $\mathcal{H}^{\lambda}$ s. The action of $\mathcal{L}$ can be extended by Leibniz rule from $\mathcal{F}^{\lambda}$ to $\mathcal{H}^{\lambda}$. But a problem arises. For elements of the critical strip of the algebra $\mathcal{L}$ it might happen that it produces infinitely many contributions. The action has to be regularized (as physicists like to call it, but it is a well-defined mathematical procedure). In this way one obtains a representation of a centrally extended $\mathcal{L}$. The 
cocycle defining the central extensions comes from the regularizing procedure. It turns out that it is local. Hence, by the classification results of Section 5.4 it is the unique central extension class defined by (20) integrated over $C_{S}$.

In $\mathcal{H}^{\lambda}$ there are certain invariant subspaces, which are generated by a certain "vacuum vector". The subalgebra $\mathcal{L}_{[+]}$annihilates the vacuum, the central element and the other elements of degree zero act by multiplication with a constant and the whole representation state is generated by $\mathcal{L}_{[-]} \oplus \mathcal{L}_{[0]}$ from the vacuum.

As the function algebra $\mathcal{A}$ operates as multiplication operators on $\mathcal{H}^{\lambda}$ the above representation can be extended to the algebra $\mathcal{D}^{1}$ (see details in Ref. 4) after one passes over to central extensions. The cocycle again is local and hence, up to coboundary, it will be a certain linear combination of the 3 generating cocycles for the differential operator algebra. In fact it will be

$$
c_{\lambda}\left[\psi_{C_{S}}^{3}\right]+\frac{2 \lambda-1}{2}\left[\psi_{C_{S}}^{4}\right]-\left[\psi_{C_{S}}^{1}\right], \quad c_{\lambda}:=-2\left(6 \lambda^{2}-6 \lambda+1\right) .
$$

Here $\psi^{3}$ is the cocycle for the vector field algebra, $\psi^{1}$ the cocycle for the function algebra, and $\psi^{4}$ the mixing cocycle, see Ref. 22 for details. Note that the expression for $c_{\lambda}$ appears also in Mumford's formula relating divisors on the moduli space of curves.

For the centrally extended algebras $\widehat{\mathfrak{g}}$ in a similar way fermionic Fock space representations can be constructed, see Ref. 8 .

\section{2. $b-c$ systems}

On the space $\mathcal{H}^{\lambda}$ the forms $\mathcal{F}^{\lambda}$ act by wedging elements $f^{\lambda} \in \mathcal{F}^{\lambda}$ in front of the semi-infinite wedge form. The forms $f^{1-\lambda} \in \mathcal{F}^{1-\lambda}$ act by contracting the elements in the semi-infinite wedge forms by using the Krichever-Novikov duality pairing. Both operations create a Clifford algebra structure, which is sometimes called a $b-c$ system, see Refs. 4, 24.

\subsection{Sugawara representation}

If we have an admissible representation of the algebra $\widehat{\mathfrak{g}}$ we can define the so called Sugawara operators. Here admissible means, that the central element operates as constant $\times$ identity, and that every element $v$ in the representation space will be annihilated by the elements in $\widehat{\mathfrak{g}}$ of sufficiently high degree (the degree depends on the element $v$ ). The Sugawara operator is an infinite formal sum of operators and is constructed as the product of the current operators which are again formal infinite sum of operators. To make the product well-defined a normal ordering has to be set. It turns out that after some rescaling the operators appearing in the formal sum of the Sugawara operators give a representation of a centrally extended vector field algebra $\mathcal{L}$. The central extension is due to the appearance of the normal ordering. Again the defining cocycle is local and we know that the central extension defined by the representation is the central extension given by our geometric cocycle $\psi_{C_{S}}^{3}$. See Refs. 25, 26, 24 for details. 


\subsection{Wess-Zumino-Novikov-Witten models and $K Z$ connection}

Despite the fact, that it is a very important application, the following description is very condensed. More can be found in Refs. 8, 9, 10. Wess-Zumino-Novikov models are defined on the basis of a fixed finite-dimensional Lie algebra $\mathfrak{g}$. One considers families of representations of the affine algebras $\widehat{\mathfrak{g}}$ (which is an almost-graded central extension of $\overline{\mathfrak{g}}$ ) defined over the moduli space of Riemann surfaces of genus $g$ with $N$ marked points. The data of the moduli of the Riemann surface and the marked points enter the definition of the algebra $\widehat{\mathfrak{g}}$ and the representation. The construction of certain co-invariants yields a special vector bundle of finite rank over moduli space, called the vector bundle of conformal blocks. With the help of the Krichever Novikov vector field algebra, via the Sugawara construction, the KnizhnikZamolodchikov connection is given. It is projectively flat. An essential fact is that certain elements in the critical strip $\mathcal{L}_{[0]}$ correspond to infinitesimal deformations of the moduli and to moving the marked points.

\subsection{Geometric Deformations of the Witt and Virasoro algebra}

As the second Lie algebra cohomology of the Witt and Virasoro algebra in their adjoint module vanishes ${ }^{27}$ both are formally and infinitesimally rigid. This means that all formal (and infinitesimal) families with special fiber these algebras are equivalent to the trivial one. Nevertheless, we showed in Ref. 28 that there exists naturally defined families of Krichever-Novikov vector field algebras defined for the torus with two marked points ${ }^{5,29}$ which have as special element the Witt algebra (resp. Viraosoro algebra). All other fibers are non-isomorphic to it. Hence, these families are even locally non-trivial. This is a phenomena which can be only observed for infinite dimensional algebras. See also the case of affine Lie algebra and some general treatment in Refs. 30, 31, 32 .

\subsection{Lax operator algebras}

Recently, a new class of current type algebras appeared, the Lax operator algebras. Some of them are related to the moduli space of framed semi-stable vector bundles, see Refs. 33, 34, 35 .

\section{Appendix A. Projective and Affine Connections}

Let $\left(U_{\alpha}, z_{\alpha}\right)_{\alpha \in J}$ be a covering of the Riemann surface by holomorphic coordinates, with transition functions $z_{\beta}=f_{\beta \alpha}\left(z_{\alpha}\right)$.

A system of local (holomorphic, meromorphic) functions $R=\left(R_{\alpha}\left(z_{\alpha}\right)\right)$ is called a (holomorphic, meromorphic) projective connection if it transforms as

$$
R_{\beta}\left(z_{\beta}\right) \cdot\left(f_{\beta, \alpha}^{\prime}\right)^{2}=R_{\alpha}\left(z_{\alpha}\right)+S\left(f_{\beta, \alpha}\right), \quad \text { with } \quad S(h)=\frac{h^{\prime \prime \prime}}{h^{\prime}}-\frac{3}{2}\left(\frac{h^{\prime \prime}}{h^{\prime}}\right)^{2},
$$

the Schwartzian derivative. Here' denotes differentiation with respect to the coordinate $z_{\alpha}$. 
A system of local (holomorphic, meromorphic) functions $T=\left(T_{\alpha}\left(z_{\alpha}\right)\right)$ is called a (holomorphic, meromorphic) affine connection if it transforms as

$$
T_{\beta}\left(z_{\beta}\right) \cdot\left(f_{\beta, \alpha}^{\prime}\right)=T_{\alpha}\left(z_{\alpha}\right)+\frac{f_{\beta, \alpha}^{\prime \prime}}{f_{\beta, \alpha}^{\prime}} .
$$

Every Riemann surface admits a holomorphic projective connection $R$, and given a point $P$ then there exists always a meromorphic affine connection holomorphic outside of $P$ and having maximally a pole of order one there. ${ }^{4}$

\section{References}

1. M. Schlichenmaier, An Introduction to Riemann Surfaces, Algebraic Curves and Moduli Spaces, 1st edition (Springer 1989).

2. M. Schlichenmaier, An Introduction to Riemann Surfaces, Algebraic Curves and Moduli Spaces, 2nd enlarged edition (1st edition published 1989, Springer, 2007).

3. I. M. Krichever and S. P. Novikov, Funkt. Anal. i. Pril. 21, 46 (1987); Funkt. Anal. i. Pril. 21, 47 (1987); Funkt. Anal. i. Pril. 23, 46 (1989).

4. M. Schlichenmaier, Verallgemeinerte Krichever - Novikov Algebren und deren Darstellungen, Dissertation (University of Mannheim, June 1990).

5. Th. Deck, Phys. Lett. B 251, no. 4, 535 (1990).

6. A. Anzaldo-Meneses, J. Math. Phys. 33, no. 12, 4155 (1992).

7. A. Ruffing, Th. Deck and M. Schlichenmaier, Lett. Math. Phys. 26, 23 (1992).

8. M. Schlichenmaier and O. K. Sheinman, Uspeki Math. Naukii (Russian Math. Surv.) 54, 213 (1999).

9. M. Schlichenmaier and O. K. Sheinman, Russian Math. Surv. 59, (4), 737 (2004).

10. M. Schlichenmaier, Elements of a Global Operator Approach to Wess-ZuminoNovikov-Witten Models, in Lie theory and its applications in physics III, eds. H. D. Doebner, V. K. Dobrev, and J. Hilgert (July 1999, Clausthal), (World Scientific, 2000), pp. 204-220.

11. M. Bordemann, J. Hoppe, P. Schaller and M. Schlichenmaier, Comm. Math. Phys. 138, 209 (1991).

12. M. Bordemann, E. Meinrenken and M. Schlichenmaier, Comm. Math. Phys. 165, 281 (1994).

13. M. Schlichenmaier, Adv. in Math. Phys., Vol. 2010, 38 pages,

14. Deformations and Contractions in Mathematics and Physics, Oberwolfach Reports, Vol. 3(1), (EMS Publishing House, 2006), p. 119.

15. Deformation Methods in Mathematics and Physics, Oberwolfach Reports, Vol. 7(3), (EMS Publishing House), p. 2503.

16. A. A. Belavin, A. M. Polyakov and A. B. Zamolodchikov, Nucl. Phys. B 241, 333 (1984).

17. A. Tsuchiya, K. Ueno and Y. Yamada, Adv. Stud. Pure Math. 19, 459 (1989).

18. M. Schlichenmaier, Lett. Math. Phys. 19, 151 (1990).

19. M. Schlichenmaier, Lett. Math. Phys. 19, 327 (1990).

20. M. Schlichenmaier, Lett. Math. Phys. 20, 33 (1990).

21. M. Schlichenmaier, Differential operator algebras on compact Riemann surfaces, in Generalized Symmetries in Physics, Clausthal 1993, eds. H.-D. Doebner, V. K. Dobrev and A. G. Ushveridze, (World Scientific, Singapore, London, 1994), pp. 425-434.

22. M. Schlichenmaier, J. reine angew. Math. 559, 53 (2003).

23. M. Schlichenmaier, Moscow Math. Journal 3, 1395 (2003). 
24. M. Schlichenmaier, Zwei Anwendungen algebraisch-geometrischer Methoden in der theoretischen Physik: Berezin-Toeplitz-Quantisierung und globale Algebren der zweidimensionalen konformen Feldtheorie, Habilitation Thesis, (University of Mannheim, June 1996).

25. M. Schlichenmaier and O. K. Sheinman, Jour. of Math. Science 92, 3807 (1998), translated from Itoki Nauki i Tekhniki, Vol. 38 (1996).

26. M. Schlichenmaier, Rep. on Math. Phys. 43, 323 (1999).

27. M. Schlichenmaier, An elementary proof of the vanishing of the second cohomology of the Witt and Virasoro algebra with values in the adjoint module, arXiv:1111.6624v1, 2011.

28. A. Fialowski and M. Schlichenmaier, Comm. Contemp. Math. 5, (6), 921 (2003).

29. M. Schlichenmaier, Jour. of Math. Phys. 34, 3809 (1993).

30. A. Fialowski and M. Schlichenmaier, Comm. Math. Phys. 260, 579 (2005).

31. A. Fialowski and M. Schlichenmaier, Inter. Jour. Theor. Phys. Vol. 46, No. 11, 2708 (2007).

32. M. Schlichenmaier, Deformations of the Witt, Virasoro, and Current Algebra, in Silvestrov, et. al., Generalized Lie theory in Mathematics, Physics and Beyond, (Springer 2009), pp. 219-234.

33. I. M. Krichever and O. K. Sheinman, Funct. Anal. Appl. 41, 284 (2007).

34. M. Schlichenmaier and O. K. Sheinman, Uspheki Math. Mauk. 63:4, 131 (2008), Russ. Math. Surveys 63:4, 727 (2008).

35. M. Schlichenmaier, Banach Center Publ. 93, 129 (2011). 